\title{
THE METRIC GEOMETRY OF THE HAMMING CUBE AND APPLICATIONS
}

\author{
F. BAUDIER, D. FREEMAN, TH. SCHLUMPRECHT, AND A. ZSÁK
}

\begin{abstract}
The Lipschitz geometry of segments of the infinite Hamming cube is studied. Tight estimates on the distortion necessary to embed the segments into spaces of continuous functions on countable compact metric spaces are given. As an application, the first nontrivial lower bounds on the $C(K)$ distortion of important classes of separable Banach spaces, where $K$ is a countable compact space in the family
\end{abstract}

$$
\left\{[0, \omega],[0, \omega \cdot 2], \ldots,\left[0, \omega^{2}\right], \ldots,\left[0, \omega^{k} \cdot n\right], \ldots,\left[0, \omega^{\omega}\right]\right\},
$$

are obtained.

\section{INTRODUCTION}

1.1. Motivation and Background. Assume that one is given a Banach space $Y$ and a class $\mathcal{C}$ of metric spaces. Given an arbitrary metric space $M$ in the class $\mathcal{C}$, it is natural to study the smallest distortion achievable when trying to embed $M$ into $Y$ through a bi-Lipschitz embedding. This quite general, quantitative embedding problem is an important topic in the nonlinear geometry of Banach Spaces. When $Y$ is a Hilbert space this problem is known as estimating the Euclidean distortion of the given class. It is well recognized that being able to accurately estimate the Euclidean distortion of some specific classes of metric spaces has tremendous and far reaching applications in both mathematics and computer science. In this paper we consider the general embedding problem when $Y$ is the Banach space $C(K)$, the space of continuous functions on a compact topological space $K$. We will mainly stay in the separable world and therefore consider only compact metric spaces $K$ as well as classes $\mathcal{C}$ contained in the class $\mathcal{M}$ of separable metric spaces. The theory is clearly isometric and, although $\mathrm{c}_{0}$ is not isometric to a $C(K)$-space for any compact space $K$, embeddings into $\mathrm{c}_{0}$ are related to those into $C(K)$-spaces. Indeed $\mathrm{c}_{0}$ is a hyperplane of the space c of convergent sequences of real numbers, which can be seen as the space $C(K)$ where $K=\gamma \mathbb{N}$ is the Alexandrov-compactification (or one-point compactification) of $\mathbb{N}$. Moreover, it is easy to show that whenever $K$ is an infinite (not necessarily metrizable) compact Hausdorff space, $C(K)$ contains a subspace isometric to $c_{0}$ (see 4, Proposition 4.3.11]). We browse briefly and chronologically through a few classical and historical embedding results into $C(K)$-spaces and $\mathrm{c}_{0}$. Back in 1906, Fréchet observed [7] that every separable metric space admits an isometric embedding into the space $\ell_{\infty}(\mathbb{N})$. An easy application of the Hahn-Banach theorem gives a linear isometric embedding of every separable Banach space into $\ell_{\infty}(\mathbb{N})$. These results can actually be cast as embedding results into a $C(K)$-space.

Date: 18 March 2014.

2010 Mathematics Subject Classification. 46B20, 46B85.

Key words and phrases. countable compact metric space, Lipschitz embedding, $C(K)$ space.

The first author's research was supported by ANR PDOC, project NoLiGeA. The second author's research was supported by NSF grant DMS1332255. The third author's research was supported by NSF grant DMS1160633. The second and fourth authors were supported by the Workshop in Analysis and Probability at Texas A\&M University in 2013. The fourth author was supported by Texas A\&M University while he was Visiting Scholar there in 2014. 
Indeed $\ell_{\infty}(\mathbb{N})$ can be identified with the space $C(\beta \mathbb{N})$ where $\beta \mathbb{N}$ denotes the StoneCech compactification of $\mathbb{N}$. Note that $\beta \mathbb{N}$ is an uncountable compact space and since $\ell_{\infty}(\mathbb{N})$ is nonseparable, $\beta \mathbb{N}$ is not metrizable. The Banach-Mazur theorem [6] asserts that every separable Banach space admits an (linear) isometric embedding into the space $C[0,1]$. Note that $[0,1]$ equipped with its canonical distance is compact and hence $C[0,1]$ is separable. With the help of Fréchet's embedding, it is easily seen that every separable metric space can be isometrically embedded into $C[0,1]$. In 1974 Aharoni proved in [3] that the $\mathrm{c}_{0}^{+}$-distortion of every separable metric space is less than 6 . In that same paper he also proved that the $\mathrm{c}_{0}$-distortion of $\ell_{1}$ is at least 2. A few years later Assouad [5] showed that the $\mathrm{c}_{0}^{+}$-distortion of every separable metric space is at most 3 . The fact that there is a bi-Lipschitz embedding with distortion exactly 3 and that this value is optimal for embeddings into $\mathrm{c}_{0}^{+}$is due to Pelant [11. Finally the end of the story regarding embeddings into $\mathrm{c}_{0}$ was completed by Kalton and Lancien [9] when they constructed an embedding with distortion 2 (respectively, 1) for every separable, respectively proper metric space. Recall that a metric space is proper if all its closed balls are compact.

1.2. Notation and Definitions. Let $M$ and $N$ be two metric spaces. Define the distortion of a map $f: M \rightarrow N$ to be

$$
\operatorname{dist}(f):=\|f\|_{\text {Lip }}\left\|f^{-1}\right\|_{\text {Lip }}=\left(\sup _{x \neq y \in M} \frac{d_{N}(f(x), f(y))}{d_{M}(x, y)}\right)\left(\sup _{x \neq y \in M} \frac{d_{M}(x, y)}{d_{N}(f(x), f(y))}\right) .
$$

If the distortion of $f$ is finite, $f$ is said to be a bi-Lipschitz embedding. The convenient notation $M \underset{\text { Lip }}{\underset{f}{\longrightarrow}} N$ means that there exists a bi-Lipschitz embedding $f$ from $M$ into $N$. We are concerned with the quantitative theory, and if $\operatorname{dist}(f) \leq C$, we use the notation $M \underset{C-\text { Lip }}{\longrightarrow} N$. The parameter $c_{N}(M)=\inf \{C \geq 1: M \underset{C-\text { Lip }}{\longrightarrow} N\}$ will be referred to as the $N$-distortion of $M$.

Let $\mathcal{F}$ be a collection of metric spaces. We can define the $N$-distortion of the class $\mathcal{F}$ as follows:

$$
c_{N}(\mathcal{F})=\sup \left\{c_{N}(M): M \in \mathcal{F}\right\} .
$$

Finally, for two families $\mathcal{F}$ and $\mathcal{G}$ of metric spaces we define

$$
c_{\mathcal{G}}(\mathcal{F})=\sup _{M \in \mathcal{F}} \inf _{N \in \mathcal{G}} c_{N}(M) .
$$

As an application of our work on the metric geometry of the Hamming cube we will give nontrivial estimates on the parameter $c_{N}(\mathcal{F})$ for the following spaces and classes:

- $N=C(K)$ for some countable compact metric space $K$.

- $\mathcal{F}$ is one of the following classes:

(1) $\mathcal{M}:=\{M: M$ separable metric space $\}$

(2) $\mathcal{S B}:=\{X: X$ separable Banach space $\}$

(3) $\mathcal{C O T}:=\{X: X$ separable Banach space with nontrivial cotype $\}$

(4) $\mathcal{T} \mathcal{Y P}:=\{X: X$ separable Banach space with nontrivial type $\}$

(5) $\mathcal{S R}:=\{X: X$ separable, reflexive Banach space $\}$.

Observe that $c_{N}(\mathcal{S B})=c_{N}(\mathcal{M})$. Indeed, it is clear that $c_{N}(\mathcal{S B}) \leq c_{N}(\mathcal{M})$, and the reverse inequality follows from the fact that every separable metric space embeds isometrically into the separable Banach space $C[0,1]$.

1.3. Stratification of the Hamming cube. We define a stratification of a metric space $M$ to be a sequence $M_{1} \subset M_{2} \subset \ldots$ of subsets of $M$ such that $M=\bigcup_{k=1}^{\infty} M_{k}$. (More generally, it is a way of expressing $M$ as a direct limit of metric spaces, but this generality will not be needed here.) The sets $M_{k}$ are the segments of $M$, and the sets $M_{k} \backslash M_{k-1}$ are the layers of $M$ (where we put $M_{0}=\emptyset$ ). In this paper 
we are concerned with stratifications of the Hamming cube. The infinite Hamming cube $H_{\infty}$ is the set of all infinite sequences in $\{0,1\}$ containing only finitely many 1 s equipped with the Hamming metric $d_{H}$, where $d_{H}(\sigma, \tau)=\left|\left\{i \in \mathbb{N}: \sigma_{i} \neq \tau_{i}\right\}\right|$. It is isometric to the metric space $\Delta_{\infty}$ consisting of the set $[\mathbb{N}]^{<\omega}$ of all finite subsets of $\mathbb{N}$ equipped with the symmetric difference metric $d_{\Delta}$, where $d_{\Delta}(A, B)=|A \triangle B|$. The isometry between $\Delta_{\infty}$ and $H_{\infty}$ is the natural one identifying a set with its indicator function.

We describe two natural stratifications of the infinite Hamming cube. For $k \in \mathbb{N}$ let $H_{k}=\{0,1\}^{k}$ thought of as a subset of $H_{\infty}$ by extending elements of $H_{k}$ to infinite binary sequences with the addition of an infinite tail of $0 \mathrm{~s}$. The layers of the stratification $\left(H_{k}\right)_{k=0}^{\infty}$ are $\{\emptyset\}$ and families of sets of the form $\{A \subset \mathbb{N}: \max A=n\}$, $n \in \mathbb{N}$. The members of the second stratification are the families $\Delta_{k}=[\mathbb{N}]^{\leq k}$ of subsets of $\mathbb{N}$ of size at most $k$. The set $\Delta_{k}$ can be identified with the rooted countably infinitely branching tree of height $k$. Note, however, that the metric $d_{\triangle}$ is not the classical graph metric of a tree.

The two stratifications share some essential metric properties despite being quite different from the combinatorial and structural standpoint. For example, $\Delta_{k}$ (respectively, $H_{k}$ ) is a $2 k$-bounded (respectively, $k$-bounded), 1 -separated metric space. However, $\Delta_{k}$ is a countable non-proper metric space while $H_{k}$ is a finite metric space. The two stratifications are different in the Lipschitz category in the following sense. Two families of metric spaces $\mathcal{F}$ and $\mathcal{G}$ shall be called Lipschitz equivalent if $c_{\mathcal{F}}(\mathcal{G}) c_{\mathcal{G}}(\mathcal{F})<\infty$. The stratifications $\mathcal{O}=\left(H_{k}\right)_{k \geq 0}$ and $\mathcal{U}=\left(\Delta_{k}\right)_{k \geq 0}$ are not Lipschitz equivalent. Indeed, the embedding $\left(\sigma_{1}, \cdots, \sigma_{k}\right) \mapsto\left\{i \in\{1, \ldots, k\}: \sigma_{i}=1\right\}$ sends $H_{k}$ isometrically into $\Delta_{k}$ (i.e., $c_{\mathcal{U}}(\mathcal{O})=1$ ), however, $c_{\mathcal{O}}(\mathcal{U})=\infty$ since it is impossible to embed a single $\Delta_{k}$ bi-Lipschitzly into any $H_{i}$ because of a cardinality obstruction (assuming $k \geq 1$ of course).

Sometimes metric information about a stratification can be used to derive metric information on the stratified space and vice-versa. However, this need not be the case. As we will see $H_{\infty}$ does not embed isometrically into $\mathrm{c}_{0}$ and this will be witnessed by $\Delta_{2}$. This is in stark contrast with the fact that every $H_{k}$, as any finite metric space, embeds isometrically into $\mathrm{c}_{0}$. So in some sense $\left(\Delta_{k}\right)_{k \geq 0}$ captures more of the structure of $\Delta_{\infty}$.

1.4. Organization of the paper. From now on we will consider countable compact metric spaces and we will focus on the following nested family:

$$
[0, \omega] \subset[0, \omega \cdot 2] \subset \cdots \subset\left[0, \omega^{2}\right] \subset \cdots \subset\left[0, \omega^{\alpha} \cdot n\right] \subset \cdots \subset\left[0, \omega^{\omega}\right],
$$

where, as usual, $\omega$ is the first infinite ordinal. It is a simple fact that if compact spaces $K$ and $L$ are homeomorphic then the Banach spaces $C(K)$ and $C(L)$ are isometrically isomorphic. Note that the converse is also true by the Banach-Stone theorem. Therefore the $C(K)$-spaces arising from the nested family above are mutually non isometric Banach spaces. However, this family has the property that $C(K)$ embeds linearly isometrically into $C(L)$ whenever $K \subset L$ since then $K$ is in fact a clopen subset of $L$.

In Section 2 we estimate from above the $C(K)$-distortion of the infinite Hamming cube and its stratification $\Delta_{k}$. We will show that when $1 \leq r \leq k<\infty$, then $c_{C\left(\left[0, \omega^{r}\right]\right)}\left(\Delta_{k}\right) \leq \min \left\{\frac{k}{r}, 2\right\}$. In particular, $\Delta_{k}$ embeds isometrically into $C\left(\left[0, \omega^{k}\right]\right)$. In Section 3 we will give lower bounds. To estimate $c_{C\left(\left[0, \omega^{r}\right]\right)}\left(\Delta_{k}\right)$ from below, we exhibit a connection between a topological property of the compact space $K$ and the $C(K)$-distortion of the metric spaces $\Delta_{k}$. Roughly speaking, if the compact metric space $K$ is small in the sense of the Cantor-Bendixson derivation, then the $C(K)$-distortion of $\Delta_{k}$ cannot be too small. More precisely, we show that if the Cantor-Bendixson index of $K$ is $k \geq 2$, then $c_{C(K)}\left(\Delta_{k}\right) \geq \frac{k}{k-1}$. In Section 4 we give 
some applications concerning the parameters $c_{C(K)}(\mathcal{M}), c_{C(K)}(\mathcal{S B}), c_{C(K)}(\mathcal{C O} \mathcal{T})$ and $c_{C(K)}(\mathcal{S R})$. We conclude with a few open questions that arise naturally from our work.

\section{Low Distortion EMBEDDings OF THE HAMMing CUBE}

2.1. Embeddings of the sets $\boldsymbol{\Delta}_{\boldsymbol{k}}$. We will show, by constructing suitable biLipschitz embeddings, that when $1 \leq r \leq k<\infty$, then $c_{C\left(\left[0, \omega^{r}\right]\right)}\left(\Delta_{k}\right) \leq \min \left\{\frac{k}{r}, 2\right\}$. In particular, $\Delta_{k}$ embeds isometrically into $C\left(\left[0, \omega^{k}\right]\right)$, and hence also into $C\left(\left[0, \omega^{r}\right]\right)$ for $r \geq k$.

We will need a description of $C(K)$-spaces as tree spaces, due to Bourgain 1 . (see also 2]), which we now proceed to describe. Recall that a tree is a set $T$ with a partial order $\preccurlyeq$ such that $b_{t}=\{s \in T: s \preccurlyeq t\}$ is finite and linearly ordered by $\preccurlyeq$ for all $t \in T$. The space $\mathrm{c}_{00}(T)$ consists of all functions $x: T \rightarrow \mathbb{R}$ with $\{t \in T: x(t) \neq 0\}$ is finite. The unit vector basis $\left(e_{t}\right)_{t \in T}$ of $\mathrm{c}_{00}(T)$ consists of functions $e_{t}$ taking the value 1 at $t$ and 0 everywhere else. For $t \in T$ the functional $\beta_{t}$ is defined by summing along the branch $b_{t}$ :

$$
\beta_{t}(x)=\sum_{s \in b_{t}} x(s) \quad\left(x \in \mathrm{c}_{00}(T)\right) .
$$

We define a norm $\|\cdot\|$ on $\mathrm{c}_{00}(T)$ by letting

$$
\|x\|=\sup _{t \in T}\left|\beta_{t}(x)\right| \quad\left(x \in \mathrm{c}_{00}(T)\right) .
$$

The tree space corresponding to $T$ is the completion $S(T)$ of $\left(\mathrm{c}_{00}(T),\|\cdot\|\right)$. It is easy to verify that $\left(e_{t}\right)$ is a normalized, monotone basis of $S(T)$. Note that the branch functionals can be expressed in terms of the biorthogonal functional as follows: $\beta_{t}=\sum_{s \in b_{t}} e_{s}^{*}$. We now let $K$ be the $w^{*}$-closure in $S(T)^{*}$ of the set $\left\{\beta_{t}: t \in T\right\}$. This is a compact Hausdorff space and $0 \in K$ if and only if $T$ has infinitely many initial nodes (i.e., elements $t \in T$ for which $s \preccurlyeq t$ implies $s=t$ ). The restriction to $K$ of the canonical embedding of $S(T)$ into $S(T)^{* *}$ is an isometric isomorphism $S(T) \rightarrow C(K)$. By the Stone-Weierstrass theorem it is onto $C(K)$ if $0 \notin K$ and onto $C_{0}(K)$ (functions vanishing at 0 ) if $0 \in K$. It turns out that every $C(K)$-space with separable dual can be represented as a tree space but we will not need this result in its full generality. We will now mention the examples relevant to us.

For each $k \in \mathbb{N}$ let $T_{k}$ be the tree $\left([\mathbb{N}]^{\leq k}, \preccurlyeq\right)$, where $s \preccurlyeq t$ if and only if $s$ is an initial segment of $t$. Thus $T_{k}$ is the rooted, countably infinitely branching tree of height $k$. As usual, we identify a set $t \subset \mathbb{N}$ with the sequence $i_{1}, i_{2}, \ldots$, where $i_{1}<i_{2}<\ldots$ are the elements of $t$. So, for example, we shall write $e_{m}$ for the basis element $e_{\{m\}}$ of $S\left(T_{k}\right)$, etc. The set $\left\{\beta_{t}: t \in T_{k}\right\}$ is homeomorphic to $\left(0, \omega^{k}\right]$ (and hence to $\left.\left[0, \omega^{k}\right]\right)$ via the map $\beta_{\emptyset} \mapsto \omega^{k}$ and

$$
\left(i_{1}, \ldots, i_{r}\right) \mapsto \sum_{j=1}^{r-1} \omega^{k-j}\left(i_{j}-i_{j-1}-1\right)+\omega^{k-r}\left(i_{r}-i_{r-1}\right),
$$

for $1 \leq r \leq k, i_{1}<\cdots<i_{r}$ (and with $\left.i_{0}=0\right)$. Thus $S\left(T_{k}\right) \cong C\left(\left[0, \omega^{k}\right]\right)$. Let us now denote by $T$ the disjoint union of the trees $T_{k}$. For $s, t \in T$ we have $s \preccurlyeq t$ if and only if for some $k$ both $s$ and $t$ belong to $T_{k}$ and $s \preccurlyeq t$ in $T_{k}$. The tree space $S(T)$ is then isometrically isomorphic to $C_{0}\left(\left[0, \omega^{\omega}\right)\right)$ which of course isometrically embeds into $C\left(\left[0, \omega^{\omega}\right]\right)$. Note also that $S(T) \cong\left(\bigoplus_{k=1}^{\infty} S\left(T_{k}\right)\right)_{\mathrm{c}_{0}}$. For the rest of the paper we fix $T_{k}$ and $T$ to be trees just described.

Theorem 1. For every $1 \leq r \leq k$ there exist a map $\varphi_{k, r}: \Delta_{k} \rightarrow C\left(\left[0, \omega^{r}\right]\right)$ such that $\operatorname{dist}\left(\varphi_{k, r}\right) \leq \frac{k}{r}$. It follows that $c_{C\left(\left[0, \omega^{r}\right]\right)}\left(\Delta_{k}\right) \leq \min \left\{\frac{k}{r}, 2\right\}$. 
Proof. For each $r \in \mathbb{N}$ we define the map

$$
f_{r}: \mathbb{N} \rightarrow S\left(T_{r}\right), \quad m \mapsto-\sum_{i=1}^{m-1} e_{i}+e_{m}+2 \sum_{j=2}^{r} \sum_{\substack{i_{1}<\cdots<i_{j} \\ i_{j}=m}} e_{i_{1}, \ldots, i_{j}} .
$$

Then for $1 \leq r \leq k$ define

$$
\varphi_{k, r}: \Delta_{k} \rightarrow S\left(T_{r}\right), \quad \sigma \mapsto \sum_{m \in \sigma} f_{r}(m)
$$

Let $\sigma, \tau \in \Delta_{k}$. We will show that

$$
\frac{r}{k} d_{\triangle}(\sigma, \tau) \leq\left\|\varphi_{k, r}(\sigma)-\varphi_{k, r}(\tau)\right\| \leq d_{\triangle}(\sigma, \tau) .
$$

Let $i_{1}<\cdots<i_{s}$ and $j_{1}<\cdots<j_{t}$ be the elements of $\sigma \backslash \tau$ and $\tau \backslash \sigma$, respectively. We need to show that

$$
\frac{r}{k}(s+t) \leq\left\|f_{r}\left(i_{1}\right)+\cdots+f_{r}\left(i_{s}\right)-f_{r}\left(j_{1}\right)-\cdots-f_{r}\left(j_{t}\right)\right\| \leq(s+t) .
$$

The upper bound follows from the triangle inequality. Indeed, for each $m \in \mathbb{N}$ and for each $t \in T_{r}$, summing $f_{r}(m)$ along the branch $b_{t}$ yields the values $-1,0,1$, and hence $f_{r}(m)$ is of norm 1 . To see the lower bound, first note that we can assume without loss of generality that $1 \leq s$ and that either $t=0$ or $i_{1}<j_{1}$. We will then prove the following statement by induction on $\max \{s, t\}$. Given $s+t$ distinct positive integers $i_{1}<\cdots<i_{s}$ and $j_{1}<\cdots<j_{t}$, where $1 \leq s \leq k$ and either $t=0$ or $1 \leq t \leq k$ and $i_{1}<j_{1}$, setting

$$
g=f_{r}\left(i_{1}\right)+\cdots+f_{r}\left(i_{s}\right)-f_{r}\left(j_{1}\right)-\cdots-f_{r}\left(j_{t}\right),
$$

there is a branch functional $\beta_{\ell_{1}, \ldots, \ell_{u}}$ with $1 \leq u \leq r$ and $i_{1} \leq \ell_{1}$ such that

$$
\left|\beta_{\ell_{1}, \ldots, \ell_{u}}(g)\right|=\left|\sum_{v=1}^{u} e_{\ell_{1}, \ldots, \ell_{v}}^{*}(g)\right| \geq \frac{r}{\max \{r, s, t\}}(s+t) .
$$

This clearly implies the lower bound of $\frac{r}{k}(s+t)$ on the norm of $g$.

If $s \leq r$ or $r \leq s \leq t$, then for $u=\min \{r, s\}$ we have

$$
\begin{aligned}
\beta_{i_{1}, \ldots, i_{u}}(g) & =e_{i_{1}}^{*}\left(f_{r}\left(i_{1}\right)+\sum_{m=2}^{s} f_{r}\left(i_{m}\right)-\sum_{n=1}^{t} f_{r}\left(j_{n}\right)\right)+\sum_{v=2}^{u} e_{i_{1}, \ldots, i_{v}}^{*}\left(f_{r}\left(i_{v}\right)\right) \\
& =1-(s-1)+t+2(u-1)=-s+t+2 u .
\end{aligned}
$$

When $s \leq r$, then $-s+t+2 u=s+t$, and we are done. If $r \leq s \leq t$, then

$$
-s+t+2 u \geq 2 r=\frac{r}{\frac{1}{2}(s+t)}(s+t) \geq \frac{r}{\max \{r, s, t\}}(s+t),
$$

as required. We finally deal with the case when $r<s$ and $t<s$. Set

$$
h=f_{r}\left(i_{2}\right)+\cdots+f_{r}\left(i_{s}\right)-f_{r}\left(j_{1}\right)-\cdots-f_{r}\left(j_{t}\right) .
$$

If $t=0$ or $i_{2}<j_{1}$, then we apply the induction hypothesis to $h$, and if $j_{1}<i_{2}$, then we apply the induction hypothesis to $-h$. In either case we obtain a branch functional $\beta_{\ell_{1}, \ldots, \ell_{u}}$ such that $i_{1}<\ell_{1}$ and $\left|\beta_{\ell_{1}, \ldots, \ell_{u}}(h)\right| \geq \frac{r}{s-1}(s+t-1)$. Since $i_{1}<\ell_{1}$, we have $\beta_{\ell_{1}, \ldots, \ell_{u}}\left(f_{r}\left(i_{1}\right)\right)=0$, and it follows that

$$
\left|\beta_{\ell_{1}, \ldots, \ell_{u}}(g)\right|=\left|\beta_{\ell_{1}, \ldots, \ell_{u}}(h)\right| \geq \frac{r}{s-1}(s+t-1) \geq \frac{r}{s}(s+t) .
$$

This completes the proof that $\operatorname{dist}\left(\varphi_{k, r}\right) \leq \frac{k}{r}$. Recall that Kalton and Lancien [9] proved that every separable metric space embeds into $\mathrm{c}_{0}$ with distortion at most 2 . It follows that $c_{C\left(\left[0, \omega^{r}\right]\right)}\left(\Delta_{k}\right) \leq \min \left\{\frac{k}{r}, 2\right\}$. 
2.2. $\boldsymbol{C}\left(\left[\mathbf{0}, \boldsymbol{\omega}^{\boldsymbol{\omega}}\right]\right)$-distortion of the Hamming cube. It follows from Theorem 1 that each $\Delta_{k}$ embeds isometrically into $C\left(\left[0, \omega^{\omega}\right]\right)$. We now prove a stronger result. Recall that a set $A \subset \mathbb{N}$ is a Schreier set if $|A| \leq \min A$ (or if $A=\emptyset$ ). The Schreier family, the set of all Schreier sets, is denoted by $\mathcal{S}_{1}$. We endow $\mathcal{S}_{1}$ with the symmetric difference metric, i.e., we consider $\mathcal{S}_{1}$ as a subset of $\Delta_{\infty}$.

Theorem 2. $\left(\mathcal{S}_{1}, d_{\triangle}\right)$ embeds isometrically into $C\left(\left[0, \omega^{\omega}\right]\right)$.

Proof. Define

$$
f_{\omega}: \mathbb{N} \rightarrow S(T), \quad m \mapsto \sum_{k=1}^{m} f_{k}(m),
$$

where $f_{k}, k \in \mathbb{N}$, are the functions defined in Theorem 1] Here we identify $x \in$ $S\left(T_{k}\right)$ with the sequence in $S(T) \cong\left(\bigoplus_{k=1}^{\infty} S\left(T_{k}\right)\right)_{\mathrm{c}_{0}}$ that has $x$ in the $k^{\text {th }}$ coordinate and zero everywhere else. Thus, more precisely, $f_{\omega}(m)$ is the sequence $\left(f_{1}(m), \ldots, f_{m}(m), 0,0, \ldots\right)$. We next define

$$
\varphi_{\omega}: \mathcal{S}_{1} \rightarrow S(T), \quad \sigma \mapsto \sum_{m \in \sigma} f_{\omega}(m)
$$

and claim that this is an isometric embedding. As before, this amounts to showing that if $\sigma, \tau \in \mathcal{S}_{1}$ and $i_{1}<\cdots<i_{s}$ and $j_{1}<\cdots<j_{t}$ are the elements of $\sigma \backslash \tau$ and $\tau \backslash \sigma$, respectively, then

$$
\left\|f_{\omega}\left(i_{1}\right)+\cdots+f_{\omega}\left(i_{s}\right)-f_{\omega}\left(j_{1}\right)-\cdots-f_{\omega}\left(j_{t}\right)\right\|=s+t
$$

Setting $g=f_{\omega}\left(i_{1}\right)+\cdots+f_{\omega}\left(i_{s}\right)-f_{\omega}\left(j_{1}\right)-\cdots-f_{\omega}\left(j_{t}\right)$, we have $\|g\| \leq s+t$ by the triangle inequality. Indeed, for each $m \in \mathbb{N}$ we have

$$
\left\|f_{\omega}(m)\right\|=\max _{1 \leq k \leq m}\left\|f_{k}(m)\right\|=1 .
$$

To get the lower bound, we may assume without loss of generality that $i_{1}<j_{1}$ (or $t=0)$ and consider the $k^{\text {th }}$ component of $g$ in $S\left(T_{k}\right)$ where $k=i_{1}$. We will show that

$$
\left\|f_{k}\left(i_{1}\right)+\cdots+f_{k}\left(i_{s}\right)-f_{k}\left(j_{1}\right)-\cdots-f_{k}\left(j_{t}\right)\right\|=s+t .
$$

Note that $s \leq|\sigma| \leq \min \sigma \leq i_{1}=k$. It follows that we can get the lower bound $s+t$ by applying the branch functional $\beta_{i_{1}, \ldots, i_{s}}$ in $T_{k}$ as in the proof of Theorem 1 ,

We now turn our attention to the infinite Hamming cube. With the help of Theorem 2 we are now able to embed the infinite Hamming cube into $C\left(\left[0, \omega^{\omega}\right]\right)$ with arbitrarily small distortion. We say that $M$ embeds almost isometrically into $N$, denoted by $M \underset{\text { a.i. }}{\longrightarrow} N$, if for every $\varepsilon>0$ there exist a bi-Lipschitz embedding $f$ from $M$ into $N$ with $\operatorname{dist}(f) \leq 1+\varepsilon$.

Theorem 3. The infinite Hamming cube $\Delta_{\infty}$ embeds almost isometrically into $C\left(\left[0, \omega^{\omega}\right]\right)$.

Proof. As before, we will in fact embed into $C_{0}\left(\left[0, \omega^{\omega}\right)\right)$ which is identified with $S(T) \cong\left(\bigoplus_{k=1}^{\infty} S\left(T_{k}\right)\right)_{\mathrm{c}_{0}}$. Fix $\varepsilon>0$. Choose a sequence $0=N_{0}<N_{1}<N_{2}<\ldots$ of integers satisfying

$$
2 m \leq \varepsilon N_{m} \quad \text { for all } m \geq 0 .
$$

We next define maps $f, \varphi$ similar to $f_{\omega}, \Delta_{\omega}$ but with a different admissibility condition. It will be clear from the definition and the proof of Theorem 2 that this new map $\varphi$ will be an isometric embedding when restricted to the class of sets $\sigma$ with $|\sigma| \leq N_{\min \sigma}$. We define

$$
f: \mathbb{N} \rightarrow S(T), \quad m \mapsto \sum_{k=1}^{N_{m}} f_{k}(m),
$$


and

$$
\varphi: \Delta_{\infty} \rightarrow S(T), \quad \sigma \mapsto \sum_{m \in \sigma} f(m)
$$

Fix $\sigma, \tau \in \Delta_{\infty}$. We will show that

$$
(1-\varepsilon) d_{\triangle}(\sigma, \tau) \leq\|\varphi(\sigma)-\varphi(\tau)\| \leq d_{\triangle}(\sigma, \tau) .
$$

By the triangle inequality, we have

$$
\|\varphi(\sigma)-\varphi(\tau)\|=\left\|\sum_{m \in \sigma} f(m)-\sum_{m \in \tau} f(m)\right\| \leq \sum_{m \in \sigma \triangle \tau}\|f(m)\|=d \triangle(\sigma, \tau) .
$$

To show the lower bound, we first observe that $\sigma$ and $\tau$ can be assumed to be disjoint. Indeed, we have

$$
\varphi(\sigma)-\varphi(\tau)=\varphi(\sigma \backslash \tau)-\varphi(\tau \backslash \sigma) \quad \text { and } \quad d_{\triangle}(\sigma, \tau)=d_{\triangle}(\sigma \backslash \tau, \tau \backslash \sigma),
$$

and so we can replace $\sigma$ and $\tau$ with $\sigma \backslash \tau$ and $\tau \backslash \sigma$ if necessary.

We next choose $m, n \in \mathbb{N}$ such that

$$
N_{m-1}<|\sigma| \leq N_{m} \quad \text { and } \quad N_{n-1}<|\tau| \leq N_{n}
$$

Set $\sigma^{\prime}=\sigma \backslash\{1, \ldots, m-1\}$ and $\tau^{\prime}=\tau \backslash\{1, \ldots, n-1\}$. Since $\sigma^{\prime}$ and $\tau^{\prime}$ are admissible, we have

$$
\left\|\varphi\left(\sigma^{\prime}\right)-\varphi\left(\tau^{\prime}\right)\right\|=d_{\triangle}\left(\sigma^{\prime}, \tau^{\prime}\right) .
$$

Next, since $\sigma^{\prime}$ and $\tau^{\prime}$ are small perturbations of $\sigma$ and $\tau$, respectively, we have

$$
\begin{aligned}
\left|\|\varphi(\sigma)-\varphi(\tau)\|-\left\|\varphi\left(\sigma^{\prime}\right)-\varphi\left(\tau^{\prime}\right)\right\|\right| & \leq\left\|\varphi(\sigma)-\varphi\left(\sigma^{\prime}\right)\right\|+\left\|\varphi(\tau)-\varphi\left(\tau^{\prime}\right)\right\| \\
& \leq d_{\triangle}\left(\sigma, \sigma^{\prime}\right)+d_{\triangle}\left(\tau, \tau^{\prime}\right) \leq(m-1)+(n-1)
\end{aligned}
$$

and

$$
\left|d_{\triangle}(\sigma, \tau)-d_{\triangle}\left(\sigma^{\prime}, \tau^{\prime}\right)\right| \leq d_{\triangle}\left(\sigma, \sigma^{\prime}\right)+d_{\triangle}\left(\tau, \tau^{\prime}\right) \leq(m-1)+(n-1) .
$$

It follows that

$$
\begin{array}{rlrl}
\|\varphi(\sigma)-\varphi(\tau)\| & \geq\left\|\varphi\left(\sigma^{\prime}\right)-\varphi\left(\tau^{\prime}\right)\right\|-(m+n-2) & & (\text { by (15) }) \\
& =d_{\triangle}\left(\sigma^{\prime}, \tau^{\prime}\right)-(m+n-2) & & (\text { by (4) }) \\
& \geq d_{\triangle}(\sigma, \tau)-2(m+n-2) & & \text { (by (6) }) \\
& =|\sigma|+|\tau|-2(m+n-2) & \\
& =|\sigma|\left(1-\frac{2(m-1)}{|\sigma|}\right)+|\tau|\left(1-\frac{2(n-1)}{|\tau|}\right) & \\
& \geq(1-\varepsilon)(|\sigma|+|\tau|)=(1-\varepsilon) d_{\triangle}(\sigma, \tau) & & (\text { by (1) and (3) })
\end{array}
$$

as required.

Remark. An interesting question presents itself in light of the two theorems above. Does $\Delta_{\infty}$ almost isometrically embed into $\mathcal{S}_{1}$ ? A positive answer with Theorem 2 would provide another proof of Theorem 3 . 


\section{Estimating the $C(K)$-Distortion From Below}

3.1. Aharoni's lower bound observed with "metric lenses". Aharoni proved that $c_{\mathrm{c}_{0}}(\mathcal{S B}) \geq 2$, and hence $c_{\mathrm{c}_{0}}(\mathcal{M}) \geq 2$. Indeed, he showed that the separable Banach space $\ell_{1}$ does not embed into $c_{0}$ with distortion strictly less than 2 . A careful inspection of his proof shows that the proof and the statement of the result can be carried out and stated without using or even mentioning the linear structure of the Banach space $\ell_{1}$. This simple but crucial observation allows us to extend Aharoni's proof to the much more general setting of embeddings into $C(K)$-spaces.

Denote by $\widetilde{\Delta}_{2}$ the subset $\{\emptyset,\{n\},\{1, i\},\{2, j\}: n \geq 1, i \geq 2, j \geq 3\}$ of the metric space $\Delta_{2}$. The following theorem is nothing else but Aharoni's lower bound theorem reformulated in purely metric terms. For the sake of completeness we include the original proof using our notation in the hope that it will make the notation used in the proof of Theorem 6 more accessible.

Theorem 4 (Aharoni). The metric space $\widetilde{\Delta}_{2}$ does not embed into $\mathrm{c}_{0}$ with distortion strictly less than 2.

Proof. Assume that $f: \widetilde{\Delta}_{2} \rightarrow \mathrm{c}_{0}$ and $C<2$ satisfy

$$
d_{\triangle}(\sigma, \tau) \leq\|f(\sigma)-f(\tau)\| \leq C d_{\triangle}(\sigma, \tau) \quad \text { for all } \sigma, \tau \in \widetilde{\Delta}_{2} .
$$

Without loss of generality one can assume that $f(\emptyset)=0$. Let $f_{n}=e_{n}^{*} \circ f$ so that $f(\sigma)=\left(f_{n}(\sigma)\right)_{n=1}^{\infty}$ for $\sigma \in \widetilde{\Delta}_{2}$. For every $i \neq j$ in $\mathbb{N}$ define

$$
\mathcal{X}_{i, j}=\left\{n \in \mathbb{N}:\left\|f_{n}(\{i\})-f_{n}(\{j\})\right\| \geq 4-2 C\right\} .
$$

Note that these are finite sets. Moreover, for every $i, j \geq 3, i \neq j, \mathcal{X}_{1,2} \cap \mathcal{X}_{i, j} \neq \emptyset$. Indeed, we have

$$
\|f(\{1, i\})-f(\{2, j\})\| \geq d_{\triangle}(\{1, i\},\{2, j\})=4 .
$$

Hence there exists $n_{i, j} \in \mathbb{N}$ such that

$$
\left\|f_{n_{i, j}}(\{1, i\})-f_{n_{i, j}}(\{2, j\})\right\| \geq 4 .
$$

It follows that

$$
\begin{aligned}
\left|f_{n_{i, j}}(\{i\})-f_{n_{i, j}}(\{j\})\right| \geq & \left|f_{n_{i, j}}(\{1, i\})-f_{n_{i, j}}(\{2, j\})\right| \\
& -\left|f_{n_{i, j}}(\{1, i\})-f_{n_{i, j}}(\{i\})\right|-\left|f_{n_{i, j}}(\{2, j\})-f_{n_{i, j}}(\{j\})\right| \\
\geq & 4-\|f(\{1, i\})-f(\{i\})\|-\|f(\{2, j\})-f(\{j\})\| \\
\geq & 4-C d_{\triangle}(\{1, i\},\{i\})-C d_{\triangle}(\{2, j\},\{j\})=4-2 C .
\end{aligned}
$$

This proves that $n_{i, j} \in \mathcal{X}_{i, j}$. Arguing along the same lines, one gets that $n_{i, j} \in \mathcal{X}_{1,2}$ as well. Therefore $\mathcal{X}_{1,2} \cap \mathcal{X}_{i, j} \neq \emptyset$ whenever $i \neq j, i, j \geq 3$. Denote by $P$ the canonical projection from $\mathrm{c}_{0}$ onto the closed linear span $Y$ of the vectors $\left(e_{n}\right)_{n \in \mathcal{X}_{1,2}}$. We now obtain a contradiction by observing that the sequence $(\operatorname{Pf}(\{n\}))_{n=3}^{\infty}$ is a $C$-bounded and $(4-2 C)$-separated sequence in the finite-dimensional Banach space $Y$. Indeed, for every $n \geq 3$,

$$
\|P f(\{n\})\| \leq\|f(\{n\})\|=\|f(\{n\})-f(\emptyset)\| \leq C d_{\triangle}(\{n\}, \emptyset)=C,
$$

and for every $i \neq j, i, j \geq 3$, we have

$$
\begin{aligned}
\|P f(\{i\})-\operatorname{Pf}(\{j\})\| & =\sup _{n \in \mathcal{X}_{1,2}}\left|f_{n}(\{i\})-f_{n}(\{j\})\right| \\
& \geq\left|f_{n_{i, j}}(\{i\})-f_{n_{i, j}}(\{j\})\right| \\
& \geq 4-2 C>0 .
\end{aligned}
$$


3.2. Estimating the $C(K)$-distortion of $\Delta_{k}$ from below. A key ingredient in estimating from below the $C(K)$-distortion of the metric space $\Delta_{k}$ is the CantorBendixson derivation for compact spaces. We next recall the definition and a few basic properties of this derivation.

Let $K$ be a compact topological space. The Cantor-Bendixson derivative $K^{\prime}$ of $K$ is the set of all accumulation points of $K$, i.e.,

$$
K^{\prime}=K \backslash\{x \in K: x \text { is an isolated point }\} .
$$

By transfinite induction one can define derivatives $K^{(\alpha)}$ of higher order $\alpha$ as follows. We set $K^{(0)}=K$. For an ordinal $\alpha$ we let $K^{(\alpha+1)}=\left(K^{(\alpha)}\right)^{\prime}$ and, finally, for a non-zero limit ordinal $\lambda$ we define $K^{(\lambda)}=\bigcap_{\alpha<\lambda} K^{(\alpha)}$.

We gather in the next proposition some basic properties of the Cantor-Bendixson derivation.

Proposition 5. Let $K$ be a compact metric space. Then

(1) $K$ is finite $\Longleftrightarrow K^{\prime}=\emptyset \Longleftrightarrow K$ is discrete;

(2) $K$ is countable $\Longleftrightarrow \exists \alpha<\omega_{1}$ such that $K^{(\alpha)}=\emptyset$;

(3) $K$ is uncountable $\Longleftrightarrow \exists \alpha<\omega_{1}$ such that $K^{(\alpha+1)}=K^{(\alpha)} \neq \emptyset$.

For a general compact topological space $K$ the smallest ordinal $\alpha$ such that $K^{(\alpha)}=K^{(\alpha+1)}$ is called the Cantor-Bendixson index (or rank) of $K$, and we denote it by $i_{\mathrm{CB}}(K)$. For example, consider the compact space $K=\left[0, \omega^{\alpha} \cdot n\right]$, where $1 \leq \alpha<\omega_{1}$ and $1 \leq n<\omega$. Then $i_{\mathrm{CB}}(K)=\alpha+1$ and $\left|K^{(\alpha)}\right|=n$. More generally, if $K$ is a countably infinite compact metric space, then for some $1 \leq \alpha<\omega_{1}$ and $1 \leq n<\omega$ we have $i_{\mathrm{CB}}(K)=\alpha+1,\left|K^{(\alpha)}\right|=n$ and $K$ is homeomorphic to $\left[0, \omega^{\alpha} \cdot n\right]$. Thus, the Cantor-Bendixson derivation gives rise to a topological classification of countable compact metric spaces, and hence an isometric classification of $C(K)$-spaces with separable dual.

Inspired by the reformulation of Aharoni's proof in terms of a metric subset of $\Delta_{2}$ we establish a link between the $C(K)$-distortion of the sequence $\left(\Delta_{k}\right)_{k \geq 1}$ and the Cantor-Bendixson index of the compact space $K$. In Section 2 we showed that $c_{C\left(\left[0, \omega^{k-1}\right]\right)}\left(\Delta_{k}\right) \leq \frac{k}{k-1}$ for $k \geq 2$. In the remainder of this section we will show that the upper bound is tight.

Theorem 6. Let $K$ be a compact topological space and $k$ be an integer with $k \geq 2$. If $\Delta_{k}$ admits a bi-Lipschitz embedding into $C(K)$ with distortion strictly less than $\frac{k}{k-1}$, then $i_{\mathrm{CB}}(K) \geq k+1$. It follows that $c_{C\left(\left[0, \omega^{k-1}\right]\right)}\left(\Delta_{k}\right)=\frac{k}{k-1}$.

Proof. Assume that there is a function $f: \Delta_{k} \rightarrow C(K)$ and a constant $D<\frac{k}{k-1}$ such that

$$
d_{\triangle}(\sigma, \tau) \leq\|f(\sigma)-f(\tau)\| \leq D d_{\triangle}(\sigma, \tau) \quad \text { for all } \sigma, \tau \in \Delta_{k} .
$$

Set $\eta=2 k-2(k-1) D$ and observe that $\eta>0$. For distinct $i, j \in \mathbb{N}$ define

$$
\mathcal{X}_{i, j}=\{\beta \in K:|f(\{i\})(\beta)-f(\{j\})(\beta)| \geq \eta\} .
$$

Consider the following statment. For each $0 \leq s \leq k$ and for any $2(k-s)$ distinct integers $i_{1}, i_{2}, \ldots, i_{k-s}, j_{1}, j_{2}, \ldots, j_{k-s}$, we have

$$
K^{(s)} \cap \mathcal{X}_{i_{1}, j_{1}} \cap \mathcal{X}_{i_{2}, j_{2}} \cap \cdots \cap \mathcal{X}_{i_{k-s}, j_{k-s}} \neq \emptyset .
$$

We will now verify this statement by induction on $s$. The theorem will then follow by putting $s=k$.

We begin with $s=0$. Let $i_{1}, \ldots, i_{k}$ and $j_{1}, \ldots, j_{k}$ be $2 k$ distinct elements of $\mathbb{N}$. Set $\sigma=\left\{i_{1}, \ldots, i_{k}\right\}$ and $\tau=\left\{j_{1}, \ldots, j_{k}\right\}$. Since $\|f(\sigma)-f(\tau)\| \geq d_{\triangle}(\sigma, \tau)=2 k$, 
there exists $\beta \in K$ such that $|f(\sigma)(\beta)-f(\tau)(\beta)| \geq 2 k$. It follows that

$$
\begin{aligned}
\left|f\left(\left\{i_{r}\right\}\right)(\beta)-f\left(\left\{j_{r}\right\}\right)(\beta)\right| \geq & |f(\sigma)(\beta)-f(\tau)(\beta)|-\left|f(\sigma)(\beta)-f\left(\left\{i_{r}\right\}\right)(\beta)\right| \\
& -\left|f\left(\left\{j_{r}\right\}\right)(\beta)-f(\tau)(\beta)\right| \\
\geq & 2 k-\left\|f(\sigma)-f\left(\left\{i_{r}\right\}\right)\right\|-\left\|f\left(\left\{j_{r}\right\}\right)-f(\tau)\right\| \\
\geq & 2 k-D d_{\triangle}\left(\sigma,\left\{i_{r}\right\}\right)-D d_{\triangle}\left(\left\{j_{r}\right\}, \tau\right) \\
\geq & 2 k-2 D(k-1)=\eta>0
\end{aligned}
$$

for each $1 \leq r \leq k$. Thus, $\beta \in \mathcal{X}_{i_{1}, j_{1}} \cap \cdots \cap \mathcal{X}_{i_{k}, j_{k}}$.

Now assume that the statement holds for some $0 \leq s<k$. Let $i_{1}, \ldots, i_{k-s-1}$ and $j_{1}, \ldots, j_{k-s-1}$ be $2(k-s-1)$ distinct elements of $\mathbb{N}$. Let

$$
L=K^{(s)} \cap \mathcal{X}_{i_{1}, j_{1}} \cap \cdots \cap \mathcal{X}_{i_{k-s-1}, j_{k-s-1}}
$$

and $n_{0}=\max \left\{i_{1}, \ldots, i_{k-s-1}, j_{1}, \ldots, j_{k-s-1}\right\}$. Note that $L$ is a closed subset of $K$. Let us denote by $R$ the restriction operator $C(K) \rightarrow C(L)$. Note that for distinct $i, j>n_{0}$ we have $L \cap \mathcal{X}_{i, j} \neq \emptyset$ by the induction hypothesis. It follows that the functions $R f(\{i\}), i>n_{0}$, are uniformly bounded and $\eta$-separated. Indeed, we have

$$
\begin{array}{r}
\|R f(\{i\})\| \leq\|f(\{i\})\| \leq\|f(\{i\})-f(\emptyset)\|+\|f(\emptyset)\| \leq D d_{\triangle}(\{i\}, \emptyset)+\|f(\emptyset)\| \\
\leq D+\|f(\emptyset)\|,
\end{array}
$$

and for distinct $i, j>n_{0}$ we can pick $\beta \in L \cap \mathcal{X}_{i, j}$ and obtain

$$
\|R f(\{i\})-R f(\{j\})\| \geq|f(\{i\})(\beta)-f(\{j\})(\beta)| \geq \eta .
$$

We deduce that $C(L)$ must be infinite-dimensional, and hence $L$ must be infinite. Since every infinite compact space has an accumulation point, we have

$$
K^{(s+1)} \cap \mathcal{X}_{i_{1}, j_{1}} \cap \mathcal{X}_{i_{2}, j_{2}} \cap \cdots \cap \mathcal{X}_{i_{k-s-1}, j_{k-s-1}} \neq \emptyset,
$$

as required.

\section{Applications AND Open PROBlems}

Let $\left(M, d_{M}\right)$ denote an arbitrary separable metric space. Let $D_{\alpha}$ be an upper bound on $c_{C\left(\left[0, \omega^{\alpha}\right]\right)}(M)$, and consider the following self-explanatory diagram.

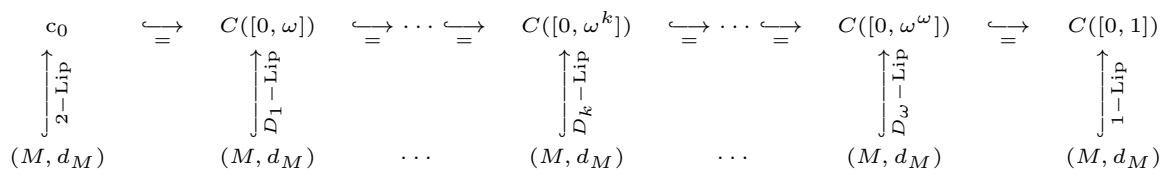

Whereas the best distortion achievable in the two extreme cases is completely understood, essentially no estimates for the values of the parameters $c_{C\left(\left[0, \omega^{\alpha}\right]\right)}(\mathcal{C})$ have been hitherto known for $\mathcal{C}$ being any class among $\mathcal{M}, \mathcal{S B}, \mathcal{C O} \mathcal{T}, \mathcal{T Y} \mathcal{P}, \mathcal{S} \mathcal{R}$ (besides the upper bound 2 which follows from Kalton-Lancien embedding result [9]). It is worth noting that since any $C(K)$-space (for $K$ countable) is $\mathrm{c}_{0}$-saturated, it cannot be a linearly isometrically universal space for the class of separable Banach spaces. Moreover, it cannot be an isometrically universal space either since Godefroy and Kalton 8 proved that if a separable Banach space $X$ embeds isometrically into a Banach space $Y$, then $Y$ contains an linear isometric copy of $X$. Our study of stratifications of the Hamming cube (Theorem $[$ ) yields nontrivial lower bounds for the first time.

Corollary 7. Let $\mathcal{C} \in\{\mathcal{M}, \mathcal{S B}, \mathcal{C O} \mathcal{T}, \mathcal{T} \mathcal{Y P}, \mathcal{S R}\}$, and let $k \in \mathbb{N}$. Then $\frac{k+1}{k} \leq$ $c_{C\left(\left[0, \omega^{k}\right]\right)}(\mathcal{C}) \leq 2$. 
Proof. We first remark that the upper bound for all $k$ is the result of Kalton and Lancien [9], and the lower bound for $k=1$ is due to Aharoni [3]. We now consider the lower bound for $k \geq 2$.

Set $K=\left[0, \omega^{k}\right]$, and note that $i_{\mathrm{CB}}(K)=k+1$. It follows from Theorem 6 that $c_{C(K)}\left(\Delta_{k+1}\right) \geq \frac{k+1}{k}$. Given $\varepsilon>0$, choose $p$ with $1<p<\infty$ such that the function $f: \Delta_{k+1} \rightarrow \ell_{p}$ defined by $f(\sigma)=\sum_{i \in \sigma} e_{i}$ is a $(1+\varepsilon)$-isometric embedding. It follows that $c_{C(K)}\left(\Delta_{k}\right) \leq(1+\varepsilon) c_{C(K)}\left(\ell_{p}\right)$. Since $\ell_{p}$ belongs to the class $\mathcal{C}$, we have $c_{C(K)}\left(\Delta_{k}\right) \leq(1+\varepsilon) c_{C(K)}(\mathcal{C})$, and the result is proved.

The following corollary is an easy consequence of Theorem 6 and the fact that $\left(\Delta_{k}\right)_{k \geq 1}$ is a stratification of $H_{\infty}$.

Corollary 8. Let $K$ be a countable compact metric space. If $H_{\infty} \underset{\text { a.i. }}{\longrightarrow} C(K)$, then $i_{\mathrm{CB}}(K) \geq \omega+1$. In particular, if $C(K)$ is an almost isometrically universal space for the class $\mathcal{C} \in\{\mathcal{M}, \mathcal{S B}, \mathcal{C O} \mathcal{T}, \mathcal{T} \mathcal{Y P}, \mathcal{S R}\}$, then $i_{\mathrm{CB}}(K) \geq \omega+1$.

Proof. It follows from Theorem [6 that $i_{\mathrm{CB}}(K) \geq k+1$ for every $k<\omega$, and hence $K^{(\omega)}=\bigcap_{k<\omega} K^{(k)} \neq \emptyset$. The result follows by Proposition [5.

Remark. Prochazka and Sánchez-González [10] using the technique of Section 3 exhibited a countable nonproper metric space which does not admit an embedding with distortion less than 2 into any $C(K)$-space with $K$ countable. Therefore for such compact spaces $K$ we have $c_{C(K)}(\mathcal{M})=c_{C(K)}(\mathcal{S B})=2$, and hence $C(K)$ cannot be an almost isometrically universal space for the classes $\mathcal{M}$ or $\mathcal{S B}$.

The following theorem, of independent interest, can also be used to prove the second part of Corollary 8 in combination with either Aharoni's original lower bound involving $\ell_{1}$ or Corollary 7 .

Theorem 9. If $\ell_{1} \underset{\text { a.i. }}{\longrightarrow} C(K)$ then $\ell_{1} \underset{\text { a.i. }}{\longrightarrow} C\left(K^{(\alpha)}\right)$ for all ordinals $\alpha<\omega$.

Proof. It is sufficient to show that if $\ell_{1} \underset{\text { a.i. }}{\longrightarrow} C(K)$, then $\ell_{1} \underset{\text { a.i. }}{\longrightarrow} C\left(K^{\prime}\right)$. Fix $\varepsilon>0$ and let $f: C(K) \rightarrow \ell_{1}$ be a function satisfying

$$
\frac{\|x-y\|_{1}}{1+\varepsilon} \leq\|f(x)-f(y)\|_{\infty} \leq\|x-y\|_{1} .
$$

Define $g: \ell_{1} \rightarrow C\left(K^{\prime}\right)$ by letting $g(x)$ be the restriction of $f(x)$ to $K^{\prime}\left(x \in \ell_{1}\right)$. We are going to show that $\operatorname{dist}(g) \leq \frac{1+\varepsilon}{1-2 \varepsilon}$, which then completes the proof.

Fix distinct vectors $x, y \in \ell_{1}$ of finite support. Let $\delta=\|x-y\|_{1}$ and $n_{0}=$ $\max \operatorname{supp}(x) \cup \operatorname{supp}(y)$. For distinct integers $i, j>n_{0}$ we have

$$
\left\|f\left(x+\delta e_{i}\right)-f\left(y+\delta e_{j}\right)\right\|_{\infty} \geq \frac{3 \delta}{1+\varepsilon} .
$$

Hence there exists $\beta \in K$ such that

$$
\left|f\left(x+\delta e_{i}\right)(\beta)-f\left(y+\delta e_{j}\right)(\beta)\right| \geq \frac{3 \delta}{1+\varepsilon} .
$$

We next observe that if (77) holds, then we also have

$$
\left|f\left(x+\delta e_{i}\right)(\beta)-f\left(x+\delta e_{j}\right)(\beta)\right| \geq \frac{(2-\varepsilon) \delta}{1+\varepsilon},
$$

and

$$
|f(x)(\beta)-f(y)(\beta)| \geq \frac{(1-2 \varepsilon) \delta}{1+\varepsilon}=\frac{(1-2 \varepsilon)\|x-y\|_{1}}{1+\varepsilon} .
$$

Now let

$$
L=\left\{\beta \in K: \exists \text { distinct } i, j>n_{o} \text { satisfying equation (77) }\right\}
$$


For $z \in \ell_{1}$ let $f_{L}(z)$ denote the restriction of $f(z)$ to $L$. By (8), the sequence $\left(f_{L}\left(x+\delta e_{i}\right)\right)_{i>n_{0}}$ in $C(L)$ is bounded and $\frac{(2-\varepsilon) \delta}{1+\varepsilon}$-separated. It follows that $L$ is infinite, and so $L \cap K^{\prime} \neq \emptyset$. By (9), for any $\beta \in L \cap K^{\prime}$ we have $|f(x)(\beta)-f(y)(\beta)| \geq$ $\frac{(1-2 \varepsilon)\|x-y\|_{1}}{1+\varepsilon}$. Thus

$$
\|g(x)-g(y)\| \geq \frac{(1-2 \varepsilon)\|x-y\|_{1}}{1+\varepsilon} .
$$

This shows that $g: \ell_{1} \rightarrow C\left(K^{\prime}\right)$ is a bi-Lipschitz embedding with constant $\frac{1+\varepsilon}{1-2 \varepsilon}$, as claimed.

We conclude by stating some open problems. In light of the above result, it is natural to ask the following.

Question 1. Does $\ell_{1}$ almost isometrically embed into $C\left(\left[0, \omega^{\omega}\right]\right)$ ?

Recall that one cannot hope for an isometric embedding because of the aformentioned result of Godefroy and Kalton [8].

Recall also that using the techniques of Theorem 6. Prochazka and SánchezGonzález [10] constructed a separable metric space $M$ for which $c_{C(K)}(M)=2$ for any (infinite) countable compact space $K$. However, it is not clear whether their example embeds into $\ell_{1}$ isometrically (or with distortion less than 2 ). Indeed, it is not known if their example isometrically embeds into any Banach space which is not already universal for $\mathcal{S B}$. So the following open problems seem to be of interest.

Question 2. Is there some non-trivial class $\mathcal{C}$ of Banach spaces and a countable compact space $K$ such that $C(K)$ is almost isometrically universal for the class $\mathcal{C}$ ?

The above question is deliberately vague. Examples we have in mind for nontrivial classes include $\mathcal{T} \mathcal{Y P}, \mathcal{C O} \mathcal{T}$ and $\mathcal{S R}$. We conclude with a more specific quantitative question.

Question 3. Let $\alpha \in\left[2, \omega_{1}\right)$. What is the exact value of $c_{C\left(\left[0, \omega^{\alpha}\right]\right)}(\mathcal{C})$ for $\mathcal{C} \in$ $\{\mathcal{T Y} \mathcal{P}, \mathcal{C O} \mathcal{T}, \mathcal{S R}\}$ ?

\section{REFERENCES}

[1] J. Bourgain, The Szlenk index and operators on $C(K)$-spaces, Bull. Soc. Math. Belg. Sér. B 31 (1979), no. 1, 87-117. MR592664 (83j:46027)

[2] E. Odell, Ordinal indices in Banach spaces, Extracta Math. 19 (2004), no. 1, 93-125. MR2072539 (2005e:46021)

[3] I. Aharoni, Every separable metric space is Lipschitz equivalent to a subset of $c_{0}^{+}$, Israel J. Math. 19 (1974), 284-291.

[4] F. Albiac and N. J. Kalton, Topics in Banach space theory, Graduate Texts in Mathematics, vol. 233, Springer, New York, 2006.

[5] P. Assouad, Remarques sur un article de Israel Aharoni sur les prolongements lipschitziens dans c 0 (Israel J. Math. 19 (1974), 284-291), Israel J. Math. 31 (1978), 97-100.

[6] S. Banach and S. Mazur, Zur Theorie der linearen Dimension, Studia Math. 4 (1933), 100112.

[7] M. Fréchet, Sur quelques points du calcul fonctionel, Rend. Circ. Mat Palermo Math. 22 (1906), 1-71.

[8] G. Godefroy and N. J. Kalton, Lipschitz-free Banach spaces, Studia Math. 159 (2003), 121141.

[9] N. J. Kalton and G. Lancien, Best constants for Lipschitz embeddings of metric spaces into $\mathrm{c}_{0}$, Fund. Mat. 3 (2008), 249-272.

[10] A. Procházka and L. Sánchez-González, Low distortion embeddings into Asplund Banach spaces, preprint.

[11] J. Pelant, Embeddings into co, Topology Appl. 57 (1994), 259-269. 
Department of Mathematics, Texas A\&M University, College Station, TX 77843 , USA and Institut de Mathématiques Jussieu-Paris Rive Gauche, Université Pierre et Marie Curie, Paris, France

E-mail address: florent@math.tamu.edu

Department of Mathematics and Computer Science, 220 N. Grand Blvd., St.Louis, MO 63103, USA

E-mail address: dfreema7@slu.edu

Department of Mathematics, Texas A\&M University, College Station, TX 77843, USA and Faculty of Electrical Engineering, Czech Technical University in Prague, Zikova 4, 166 27, Prague, Czech Republic

E-mail address: schlump@math.tamu.edu

Peterhouse, Cambridge, CB2 1RD, UK

E-mail address: a.zsak@dpmms.cam.ac.uk 